\title{
From osteoarthritic synovium to synovial- derived cells characterization: synovial macrophages are key effector cells
}

\author{
Cristina Manferdini ${ }^{1,2}$, Francesca Paolella ${ }^{1}$, Elena Gabusi ${ }^{2}$, Ylenia Silvestri1, Laura Gambari ${ }^{1}$, Luca Cattini ${ }^{1}$, \\ Giuseppe Filardo ${ }^{3}$, Sandrine Fleury-Cappellesso ${ }^{4}$ and Gina Lisignoli ${ }^{1,2^{*}}$
}

\begin{abstract}
Background: The aim of the study was to characterize synovial cells from OA synovium with low-grade and moderate-grade synovitis and to define the role of synovial macrophages in cell culture.

Methods: Synovial tissue explants were analyzed for the expression of typical markers of synovial fibroblasts (SF), synovial macrophages (SM) and endothelial cells. Synovial cells at passage 1 (p.1) and 5 (p.5) were analyzed for different phenotypical markers by flow cytometric analysis, inflammatory factors by multiplex immunoassay, anabolic and degradative factors by qRT-PCR. P.1 and p.5 synovial cells as different cell models were co-cultured with adipose stem cells (ASC) to define SM effects.

Results: Synovial tissue showed a higher percentage of CD68 marker in moderate compared with low-grade synovitis. Isolated synovial cells at p.1 were positive to typical markers of SM (CD14, CD16, CD68, CD80 and CD163) and SF (CD55, CD73, CD90, CD105, CD106), whereas p.5 synovial cells were positive only to SF markers and showed a higher percentage of CD55 and CD106. At p.1 synovial cells released a significantly higher amount of all inflammatory (IL6, CXCL8, CCL2, CCL3, CCL5) and some anabolic (IL10) factors than those of p.5. Moreover, p.1 synovial cells also expressed a higher amount of some degradative factors (MMP13, S100A8, S100A9) than p.5 synovial cells. Co-culture experiments showed that the amount of SM in p.1 synovial cells differently induced or down-modulated some of the inflammatory (IL6, CXCL8, CCL2, CCL3, CCL5) and degradative factors (ADAMTS5, MMP13, S100A8, S100A9).

Conclusions: We found that p.1 (mix of SM and SF) and p.5 (only SF) synovial cells represent two cell models that effectively reproduce the low- or moderate-grade synovitis environment. The presence of SM in culture specifically induces the modulation of the different factors analyzed, confirming that SM are key effector cells.
\end{abstract}

Keywords: Osteoarthritis, Synovial fibroblasts, Synovial macrophages, Inflammatory factors, Degradative factors

\section{Background}

Osteoarthritis (OA) is defined as a disease of the whole joint because it affects not only the cartilage but the subchondral bone and the synovial tissue that undergo structural and metabolic modifications [1, 2]. Different reports have recognized the importance of synovial inflammation as a key factor associated with the pain and symptoms of

\footnotetext{
* Correspondence: gina.lisignoli@ior.it

${ }^{1} \mathrm{SC}$ Laboratorio di Immunoreumatologia e Rigenerazione Tissutale, Istituto Ortopedico Rizzoli, Via di Barbiano 1/10, Bologna 40136, Italy

${ }^{2}$ SD Laboratorio RAMSES, Istituto Ortopedico Rizzoli, Bologna 40136, Italy Full list of author information is available at the end of the article
}

OA, even in the early phase of the disease [3-5]. New imaging techniques (ultrasound and magnetic resonance imaging) demonstrate synovitis with effusion in $95 \%$ of patients with OA and synovitis without effusion in $70 \%$ of patients [6]. A recent report has identified a gene expression pattern of cells from inflamed and non-inflamed areas of synovial tissue in OA [7].

Synovial inflammation is a process characterized by synovial thickening (hypertrophy and hyperplasia) and cell infiltration (lymphocytes and macrophages) $[8,9]$. Histological analysis of synovium in OA shows an increased number of lining cells and infiltrating cells, mainly 
consisting of macrophages $[8,10]$ with a very low percentage of B and T cells [11]. Synovial inflammation is now accepted as an important feature of the symptoms and progression of OA [6].

Normal synovial layers in OA are composed of synovial fibroblasts (SF) and inflammatory leukocytes (lymphocytes and macrophages) [12]. SF are mesenchymal cells that display many characteristics of fibroblasts, including vimentin, CD55, CD90, cadherin-11, vascular adhesion molecule-1 (VCAM-1) and intracellular adhesion molecule-1 (ICAM-1) [13-15]. SF constitutively produce IL6, chemokine (C-X-C motif) ligand (CXCL)8/ IL8, chemokine (C-C motif) ligand (CCL)2/monocyte chemotactic protein (MCP-1), transforming growth factor (TGF) $\beta$, and fibroblast growth factor [13]. Moreover, synovium was recently reported to contain cells that, after isolation and cell-culture expansion, display a mesenchymal stem cell (MSC) phenotype indistinguishable from SF [15]. Synovial macrophage-like (SM) cells in OA show a phenotype similar to other resident cell macrophages, including CD11b, CD14, CD16 and CD68, and they produce the main inflammatory mediators, such as IL1, IL6, TNF $\alpha$, matrix metalloproteinases (MMPs) and aggrecanases (ADAMTS), which contribute to articular matrix degradation [16]. Isolated synovial cells in OA are mainly composed of SF with 7 \% SM, less than $0.5 \%$ neutrophils and less than $0.1 \% \mathrm{~T}$ cells [17]. It has been shown that depletion of CD14-positive SM results in a decline in IL1 $\beta$ and TNF $\alpha$, thus indicating that these cells play a role in inflammation [17]. In the early stage of $\mathrm{OA}$, a unique chemokine signature has been associated with synovial inflammation [3, 18]. CCL5/RANTES and CCL19/macrophage inflammatory protein (MIP) $3 \beta$ chemokines are mainly associated with inflammation [19].

Up to now there has been no in-depth characterization of the synovium and isolated synovial cells in OA. Recent papers $[20,21]$ have highlighted the importance of better characterization of synovial cells to elucidate the relationship between the different cell types to better define an in vitro cell model. This characterization might lead to better understanding of the interplay between cells in inflammatory and non-inflammatory conditions, to define a synovial cell model as a foundation for devising tailored therapeutic intervention.

To gain new insight into this topic we first analyzed synovial tissue biopsies for the expression of typical markers of SF, SM and endothelial cells and then we followed their expression in isolated synovial cells both at passage 1 (mix of SF and SM), and passage 5 (SF). Subsequently, we performed in-depth analysis of isolated cells for different phenotypical markers, inflammatory, anabolic and degradative factors. Finally, as inflammation induces adipose stem cells (ASC) to exert antiinflammatory effects [22], we used these cells to test whether in co-culture experiments the presence of SM in synovial cells differently induced or down-modulated some of the inflammatory (IL6, CXCL8/IL8, CCL2/ MCP-1, CCL3/MIP1- $\alpha$, CCL5/RANTES) and degradative factors (ADAMTS5, MMP13, S100A8, S100A9) analyzed. We found that SM in culture induces the specific modulation of the different factors analyzed, thus confirming that SM are key effector cells.

\section{Methods}

\section{Patient characterization}

Synovial tissues were obtained from 26 patients with OA (14 women and 12 men, mean age $66 \pm 11.10$ years, body mass index $28 \pm 4.45 \mathrm{Kg} / \mathrm{m}^{2}$, disease duration $7 \pm 4.8$ years) and Kellgren/Lawrence grade 3/4 [23], who were undergoing total knee replacement surgery. Subcutaneous abdominal fat was obtained from six healthy patients undergoing liposuction. The study was approved by the Rizzoli Orthopaedic Institute ethical committee and all patients provided informed consent (Protocol number 15274).

\section{Synovial tissue characterization}

Synovial tissue specimens were fixed in B5 solution (freshly prepared 9:1 mixture of mercuric-chloride/40 \% formaldehyde) at room temperature for $2 \mathrm{~h}$ and embedded in paraffin, and serial tissue sections ( $4 \mu \mathrm{m}$ thick) from each specimen were prepared and routinely stained with hematoxylin-eosin. The histopathological features of each synovial tissue specimen were evaluated according to the synovitis inflammation scoring system described by Krenn [24], which rank each of the alteration evaluated (hyperplasia of the synovial lining layer, inflammatory infiltrate and stromal cell density) on a scale from 0 to 3 . The parameters of synovitis inflammation scoring system were summarized as follows: $0-1$ no synovitis; 2-3 low-grade synovitis; 4-6 moderate-grade synovitis; and 7-9 high-grade synovitis. The scoring was performed by two independent observers (CM and $\mathrm{GL})$.

\section{Immunohistochemical analysis of synovial tissue}

Serial sections were incubated overnight at $4{ }^{\circ} \mathrm{C}$ with monoclonal anti-human-CD55 $(2.5 \mu \mathrm{g} / \mathrm{ml}$, Millipore, Temecula, CA, USA), -CD68 (10 $\mu \mathrm{g} / \mathrm{ml}$, Dako Cytomation, Denmark), -Factor VIII (10 $\mu \mathrm{g} / \mathrm{ml}$ Dako), -CCL3/ MIP1 $\alpha(2.5 \mu \mathrm{g} / \mathrm{ml}$ R\&D Systems, Minneapolis, MN, USA) and -S100A8 $(4.5 \mu \mathrm{g} / \mathrm{ml} \mathrm{R \& D})$ diluted in Tris-buffered saline (TBS) containing $0.1 \%$ bovine serum albumin (BSA). Samples were then rinsed in TBS and sequentially incubated at room temperature for 20 minutes with multilinker biotinylated secondary antibody (Biocare Medical, Walnut Creek, CA, USA) and alkaline phosphataseconjugated streptavidin (Biocare Medical). The reactions were developed using fast red substrate (Biocare Medical), counterstained with hematoxylin, and mounted in glycerol 
gel. Negative controls were performed using isotype control (Dako Cytomation). Semiquantitative analysis of immunohistochemically stained slides were performed on 20 microscopic fields $(\times 200$ magnification $)$ for each section. The analysis was performed using Red/Green/Blue (RGB) with Software NIS-Elements and Eclipse 90i microscope (Nikon Instruments Europe BV). Briefly, we acquired the total number of blue-stained nuclei and the total number of positive-stained red cells. The data were expressed as percentage of positive cells for CD55 and CD68, respectively. For Factor VIII analysis we counted the number of positive vessels in 20 microscopic fields. The data were expressed as the mean number of positive vessels $/ 5 \mathrm{~mm}^{2}$ area.

\section{Isolation and characterization of synovial cells from non- digested fragments}

Synovial cells were isolated following a standardized procedure as previously described [25] and were used for the experiments at both passages 1 and 5 . Briefly, synovial tissue was fragmented and the pieces cultured for 7 days. Cultured synovial fragments were removed and fixed as described above. Synovial cells were grown in OPTIMEM culture medium (Life Technologies Italia, Monza, Italy) supplemented with $100 \mathrm{U} / \mathrm{ml}$ penicillin and $100 \mu \mathrm{g} / \mathrm{ml}$ streptomycin in a humidified atmosphere, at $37{ }^{\circ} \mathrm{C}$ with $5 \% \mathrm{CO}_{2}$.

Synovial cells at both passages 1 (p.1) and 5 (p.5) were characterized by flow cytometry using the following markers expressed by SF (CD55 (2.5 $\mu \mathrm{g} / \mathrm{ml}$, Millipore), CD73, CD90, and CD105 (5 $\mu \mathrm{g} / \mathrm{ml}, \mathrm{BD}$ Pharmingen, San Jose, CA, USA), CD106 (10 $\mu \mathrm{g} / \mathrm{ml}$, Millipore), SM (CD14 and CD16 (5 $\mu \mathrm{g} / \mathrm{ml}$, Dako), CD68 (5 $\mu \mathrm{g} / \mathrm{ml}, \mathrm{BD}), \mathrm{CD} 80$ (2 $\mu \mathrm{g} / \mathrm{ml}$, GeneTex Inc., Irvine, CA, USA), and CD163 $(10 \mu \mathrm{g} / \mathrm{ml}$, Abcam, Cambridge, UK)), and by endothelial (CD31, $2 \mu \mathrm{g} / \mathrm{ml}, \mathrm{R} \& \mathrm{D}$ ) and mononuclear cells (CD3, CD34, and CD45 (5 $\mu \mathrm{g} / \mathrm{ml}$, Dako). Briefly, after harvesting cells upon detachment, they were washed twice with PBS, centrifuged, and washed in a flow cytometry buffer (PBS supplemented with $2 \%$ BSA and $0.1 \%$ sodium azide).

Aliquots of $1 \times 10^{5}$ cells were then incubated with primary antibodies at $4{ }^{\circ} \mathrm{C}$ for 30 minutes, washed twice with a flow cytometry buffer, and incubated with polyclonal rabbit anti-mouse immunoglobulins/fluorescein isothiocyanate (FITC) conjugate (Dako Cytomation) at $4{ }^{\circ} \mathrm{C}$ for 30 minutes. After two final washes, the cells were analyzed using a fluorescence-activated cell sorting (FACS) CantoII Cytometer (Becton Dickinson). For isotype control, non-specific mouse IgG was substituted for the primary antibody.

\section{Passage 1 and 5 synovial cells as specific cell models for cell co-culture}

Synovial cells at both passages 1 and 5 (100,000 cells/ well) were seeded in the lower chamber of a 6 -well plate and co-cultured with clinical grade ASC (100,000 cells in Transwells ${ }^{\circ}$ ) for 7 days (medium was changed at day 2) in complete DMEM using a defined cell ratio (1:1) that assures no cell proliferation, as we previously reported [22]. ASC were isolated from subcutaneous abdominal fat according to Good Manufacturing Practice (GMP) [26], and were grown in $\alpha M E M$ supplemented with platelet lysate (PLP) and characterized for the $C D$ markers CD14, CD34, CD45, CD73, CD90 (5 $\mu \mathrm{g} / \mathrm{ml}$, BD Pharmingen) and CD13 (1 $\mu \mathrm{g} / \mathrm{ml}$, eBioscience, San Diego, CA, USA) as we previously described [22, 26] (data not shown). Control cells were mono-cultures of ASC and synovial cells at both passages 1 and 5 . The cells were harvested on day 7 for quantitative RT-PCR analysis and supernatant stored at $-80{ }^{\circ} \mathrm{C}$. The concentrations of IL6, CXCL8/IL8, CCL2/MCP-1, CCL3/MIP1$\alpha$, and CCL5/RANTES were analyzed in the supernatant for all conditions tested as described above.

\section{Cytokine and chemokine release in supernatants}

The concentrations of IL1 $\beta$, IL4, IL6, CXCL8/IL8, IL10, CCL2/MCP-1, CCL3/MIP1 $\alpha$, CCL5/RANTES, TGF $\beta$, and TNFo were simultaneously evaluated in the supernatants of ASC, synovial cells at both passages (1 and 5) in mono- and co-cultures, using multiplex bead-based sandwich immunoassay kits (BioRad Laboratories Inc., Segrate, Italy) following the manufacturer's instructions. Briefly, we added $50 \mu \mathrm{l}$ to each well of the diluted standards (fourfold dilution series), controls, and samples in triplicate and added $50 \mu \mathrm{l}$ of coupled beads, and the plate was incubated at room temperature for 30 minutes. The plate was then washed three times with $100 \mu \mathrm{l}$ of wash buffer and incubated with $25 \mu \mathrm{l}$ of detection antibodies for 30 minutes. Finally, the plate was washed three times and incubated with $50 \mu \mathrm{l}$ of streptavidin-PE for 30 minutes and measured in a reader (Luminex Bioplex system, Bio-Rad Laboratories Inc.).

\section{Real-time quantitative reverse transcription polymerase chain reaction (qRT-PCR) analysis}

Total RNA was extracted from human ASC, synovial cells in mono- and co-cultures, using RNA PURE reagent (Euroclone Spa, Pero, Italy) according to the manufacturer's instructions, and then was treated with DNase I (DNA-free Kit, Life Technologies). Reverse transcription was performed using SuperScript VILO (Life Technology) reverse transcriptase and random hexamers, following the manufacturer's protocol.

Forward and reverse oligonucleotides for PCR amplification of ADAMTS4, ADAMTS5, MMP13, S100A8, and S100A9 are described in Table 1, and real-time PCR was run as previously described [22]. All primer efficiencies were confirmed to be high ( $>90 \%)$ and comparable (Table 1). For each target gene, messenger RNA (mRNA) 
Table 1 Oligonucleotide primers used for real-time polymerase chain reaction

\begin{tabular}{llllc}
\hline Target gene & Primers (forward and reverse) & Product size (bp) & GenBank accession number & Primer efficiency (\%) \\
\hline RPS9 & GATTACATCCTGGGCCTGAA ATGAAGGACGGGATGTTCAC & 161 & NM_001013 & 94.5 \\
ADAMTS4 & CTGCCTACAACCACCG GCAACCAGAACCGTCC & 293 & NM_005099.4 & 99.1 \\
ADAMTS5 & GCACTTCAGCCACCATCAC AGGCGAGCACAGACATCC & 187 & NM_007038.3 & 92.4 \\
MMP13 & TCACGATGGCATTGCT GCCGGTGTAGGTGTAGA & 277 & NM_002427 & 94.5 \\
S100A8 & TAGAGACCGAGTGTCCTCA CGCCCATCTTATCACCAGA & 126 & NM_002964.4 & 93.4 \\
S100A9 & CCATCATCAACACCTTCCACCA CTGCTTGTCTGCATTTGTTCC & 179 & NM_002965.3 & 91.4 \\
\hline
\end{tabular}

levels were calculated, normalized to RPS9 according to the formula $2^{-\Delta \mathrm{Ct}}$, and expressed as a percentage of the reference gene, as this was expressed in the same amount in all conditions tested.

\section{Statistical analysis}

Statistical analysis was performed using non-parametric tests because the data did not have a normal distribution (Kolmogorov-Smirnov test). Friedman's analysis and Dunn's post hoc test was used to analyze more than two groups of paired data, the Mann-Whitney $U$ test was used to analyze unpaired two-group data and the Wilcoxon test was used to analyze paired two-group data. Groups with small samples were evaluated using the exact method. Values were expressed as the median and interquartile range. CSS Statistica Statistical Software (Statsoft Inc., Tulsa, OK, USA) was used for analysis and values of $p<0.05$ were considered significant.

\section{Results}

\section{OA synovial tissue explant characterization}

Synovial tissue explants from 26 patients with OA were first scored on hematoxylin-eosin-stained slides, as reported by Krenn [24] and we found low-grade synovitis in 4 samples and moderate-grade synovitis in 22 samples. Vessel proliferation was also evaluated on Factor VIII-stained slides and there were fewer positive vessels $\left(90 \pm 35 / 5 \mathrm{~mm}^{2}\right.$ area $)$ in low-grade than in moderategrade synovitis $\left(222 \pm 79 / 5 \mathrm{~mm}^{2}\right.$ area). Then, for indepth analysis of the main synovial cell populations present in the synovial tissue, we analyzed CD68 and CD55 to establish the percentage of synovial macrophages and synovial fibroblasts, in both low- and moderate-grade synovitis. As shown in Fig. 1a, CD68 was mainly positive on synovial macrophages located in the lining layer and on a few in the sublining layer (Additional file 1). There were approximately $13 \%$ and $27 \%$ of CD68-positive cells in low- and moderategrade synovitis, respectively (Fig. 1b). The CD55 typical marker of synovial fibroblast was positive both on the sublining and lining layers (Fig. 1a) (Additional file 1) and was approximately $70 \%$ positive in both low- and moderategrade synovitis (Fig. 1b).

\section{Synovial cells characterization}

The cells outgrowing from cultured-synovium tissue fragments (Fig. 2a) were then morphologically and phenotypically analyzed. As shown in Fig. $2 \mathrm{a}$, at p.0 cells started outgrowth from synovial tissue fragments; at p.1 we found two cell types, one spindle-shaped (defined as synovial fibroblasts, SF) and one with polygonal-star morphology (defined as synovial macrophages, SM), whereas at p.5 all cells had only spindle-shaped morphology. Moreover, to confirm that SM and SF had these peculiar cell morphologies, we immunocytochemically stained the isolated p.1 and p.5 cells with anti-CD68 and anti-CD55, typical markers of SM and SF, respectively (data not shown).

These cells at both passages (p.1 and p.5), were then characterized by flow cytometry for markers expressed by SF (CD55, CD73, CD90, CD105, and CD106), SM (CD14, CD16, CD68, CD80, and CD163), endothelial cells (CD31), and mononuclear cells (CD3, CD34, and CD45). As shown in Fig. 2b, p.1 synovial cells had a very low percentage $(<3 \%)$ of CD3, CD31, CD34, and CD45, an intermediate percentage (10-20\%) of CD14, CD16, CD68, CD80, CD106 and CD163, and a high percentage (60-100\%) of CD55, CD73, CD90, and CD105. Interestingly, CD80 and CD163 were expressed (approximately $12 \%)$ only by p. 1 synovial cells. Conversely, p. 5 synovial cells had a very low or negative percentage of all the markers analyzed except for CD55, CD73, CD90, CD105 and CD106. In particular, CD55 and CD106 were the only markers more highly expressed by p.5 synovial cells.

\section{Factors released by OA synovial cells}

We subsequently evaluated inflammatory factors (IL1 $\beta$, TNF $\alpha$, IL6, CXCL8/IL8, CCL2/MCP-1, CCL3/MIP1 $\alpha$, and CCL5/RANTES) and anabolic factors (TGF $\beta$, IL4, and IL10) released by p.1 and p.5 OA synovial cells. As shown in Fig. 3, p.1 synovial cells produced significantly more IL6, CXCL8/IL8, CCL2/MCP-1, CCL3/MIP1 $\alpha$, CCL5/RANTES, and IL10 than those at p.5. IL1 $\beta$, TNF $\alpha$, TGF $\beta$ and IL4 were not detected at either passage (p.1 or p.5). In particular, p.1 synovial cells released more IL6, CXCL8/IL8, and CCL2/MCP-1 than CCL3/MIP1 $\alpha$, CCL5/RANTES, and IL10. Interestingly, CCL2/MCP-1 

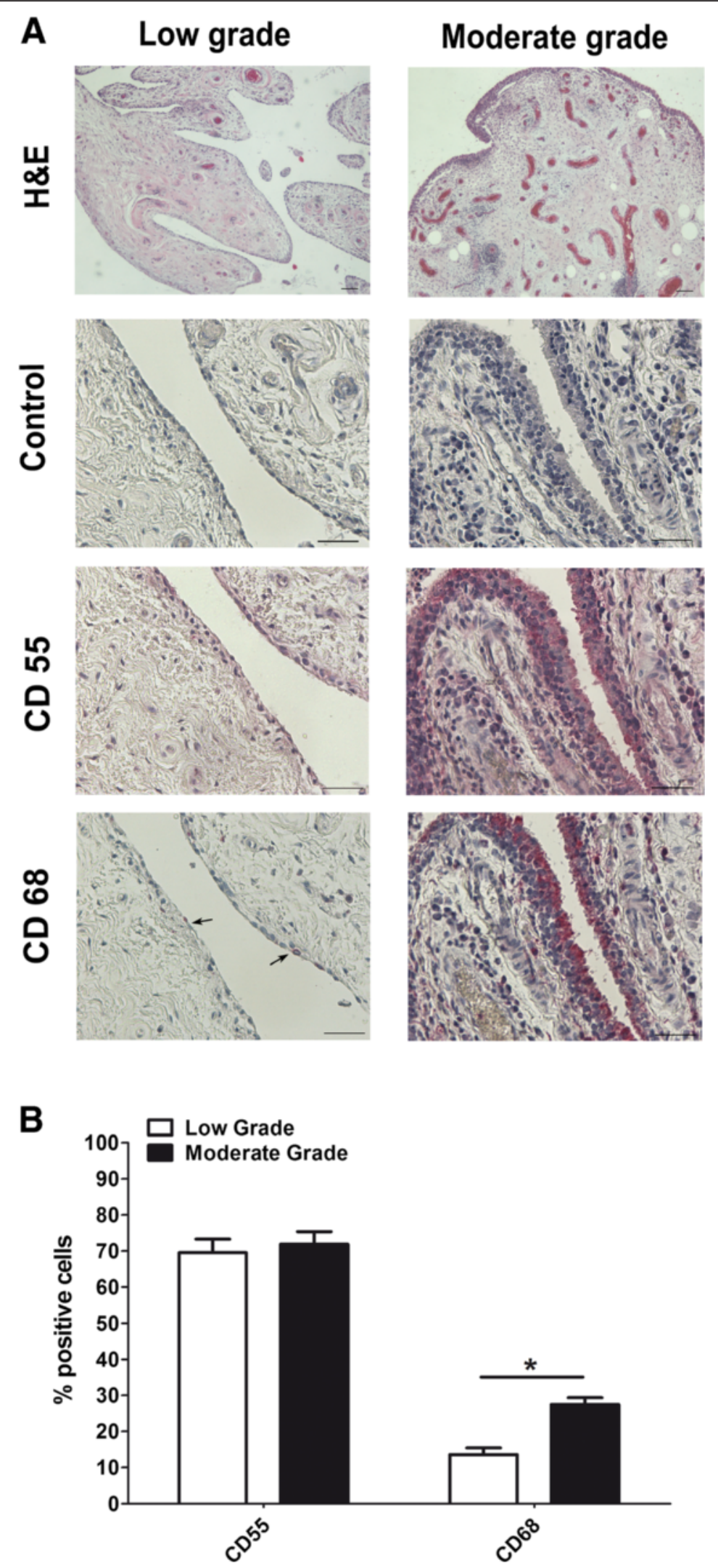

Fig. 1 (See legend on next page.) 
(See figure on previous page.)

Fig. 1 Characterization of synovial tissue in osteoarthritis $(\mathrm{OA})$. a Representative samples with low-grade (left) and moderate-grade (right) OA synovitis, stained with hematoxylin-eosin (H\&E). Bars $100 \mu \mathrm{m}$ (magnification × 40). Immunohistochemical analysis of CD55 and CD68 on representative cases with low-grade (left) and moderate-grade (right) synovitis in OA. Negative control for CD55 and CD68 (Contro). Bars $50 \mu \mathrm{m}$. b Percentage of positive cells to CD55 and CD68 analyzed in both low-grade $(n=4)$ and moderate-grade $(n=22)$ synovitis in OA. Data are expressed as the median and interquartile range. *Significant differences between low-grade and moderate-grade synovitis: $p<0.005$

was the most abundant factor released by p.5 synovial cells, whereas there was less IL6, CXCL8/IL8, and CCL5/ RANTES. IL10 and CCL3/MIP1 $\alpha$ from p.5 synovial cells were at the limit of detection or not detected, respectively.

\section{OA synovial cell degradative factors}

Then we analyzed different factors (ADAMTS4, ADAMTS5, MMP13, S100A8, S100A9) involved in the degradation of joint tissue. As shown in Fig. 4, both synovial cells at p.1 and p.5 expressed the same level of
ADAMTS4, whereas there was significantly more ADAMTS5 expressed in p.5 than in p.1 synovial cells. Conversely, MMP13, S100A8, and S100A9 were highly expressed in p. 1 synovial cells, but there was very low expression of in MMP13 in p.5 synovial cells, and S100A8 and S100A9 were not detected.

Synovial macrophages influence cell co-culture effects

The presence of SM in p.1 synovial cells significantly increased the release of inflammatory, anabolic and

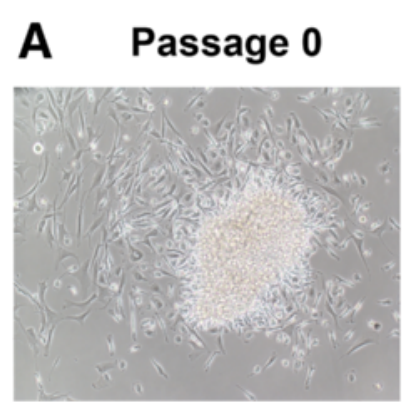

Passage 1

Passage 5

B
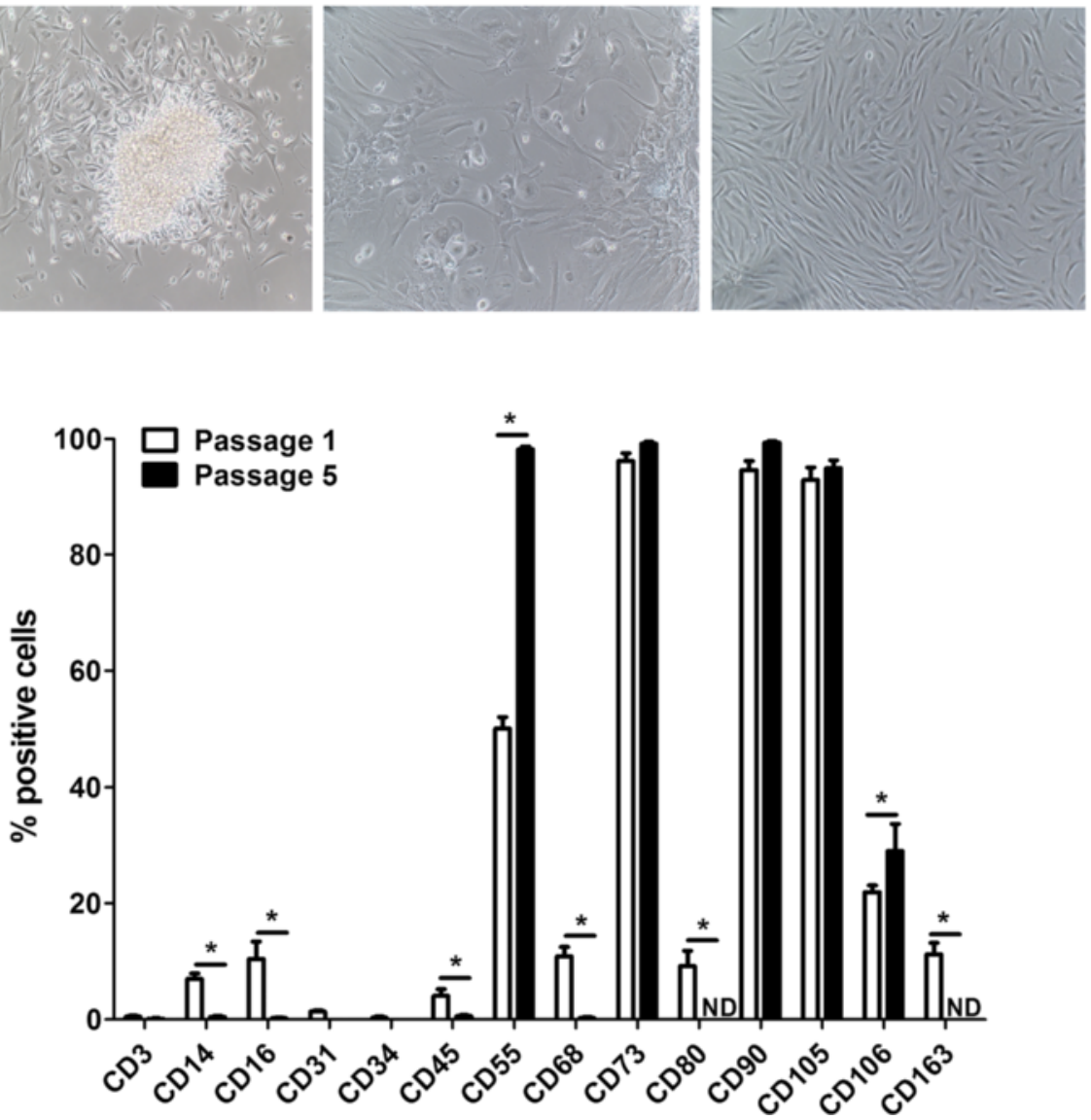

Fig. 2 Evaluation of isolated passage 1 and passage 5 synovial cells from moderate-grade synovitis in osteoarthritis. a Outgrowth of synovial cells from synovial non-digested fragments (Passage 0). Passage 1 synovial cells characterized by a mix of cells with a spindle and a polygonal-star shape. Passage 5 synovial cells characterized only by spindle-shaped morphology. b CD3,CD14, CD16, CD31, CD34, CD45, CD55, CD68, CD73, CD80, CD90, CD105, CD106, and CD163 immunocytochemical staining on passage 1 and passage 5 synovial cells analyzed by flow cytometry. Data are expressed as the median and interquartile range $(n=22)$. *Significant differences between passage 1 and passage 5 synovial cells: $p<0.005$. ND not detected 


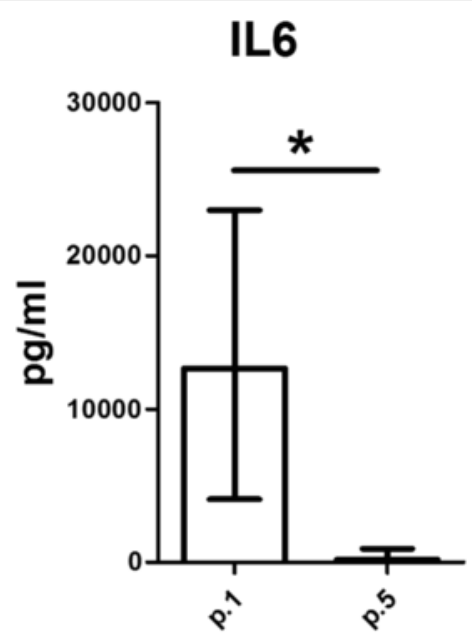

\section{CXCL8/IL8}

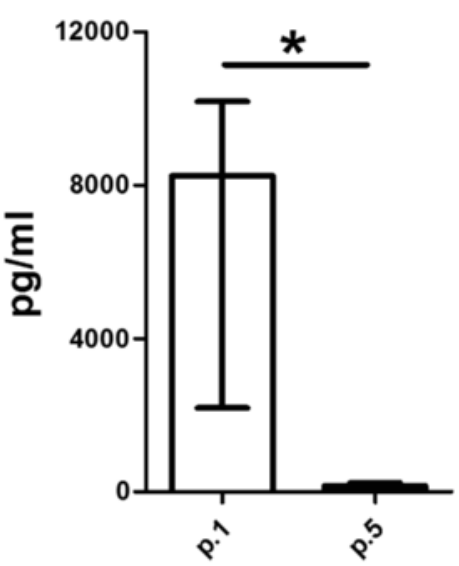

CCL2/MCP1

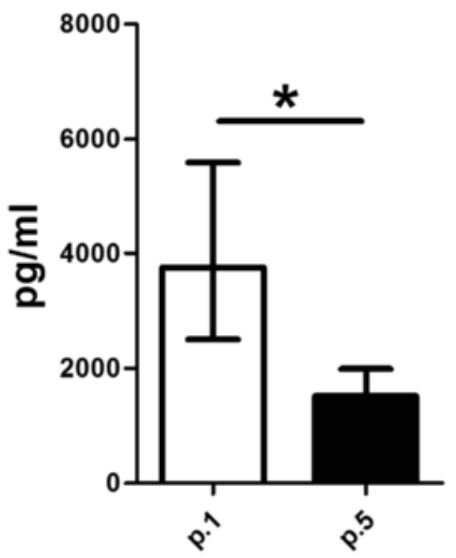

CCL3/MIP1 $\alpha$

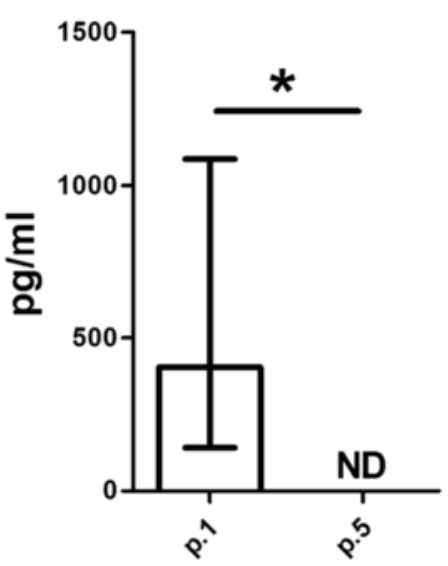

CCL5/RANTES
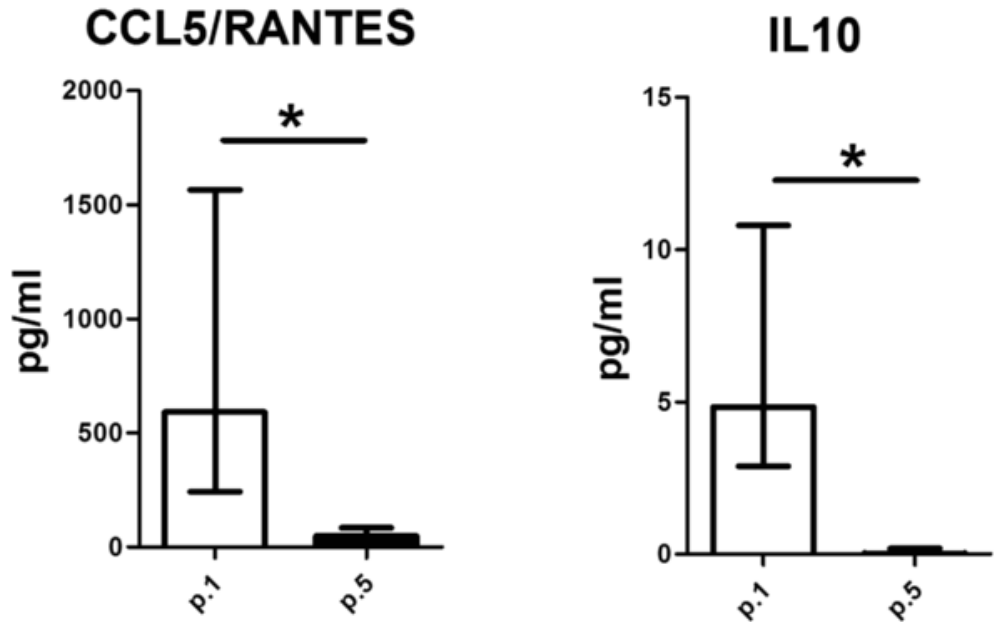

Fig. 3 Evaluation of inflammatory and anabolic factors released by passage $1(p .1)$ and passage 5 (p.5) synovial cells from osteoarthritic moderategrade synovitis. IL6, CXCL8/IL8, CCL2/monocyte chemotactic protein 1 (MCP-1), CCL3/macrophage inflammatory protein 1a (MIP1a), CCL5/RANTES, and IL 10 were evaluated in the supernatant of both p.1 and p.5 synovial cells as described in "Methods". Data are expressed as the median and interquartile range $(n=22)$. ${ }^{*}$ Significant differences between synoviocytes at p.1 and p.5: $p<0.005$ 


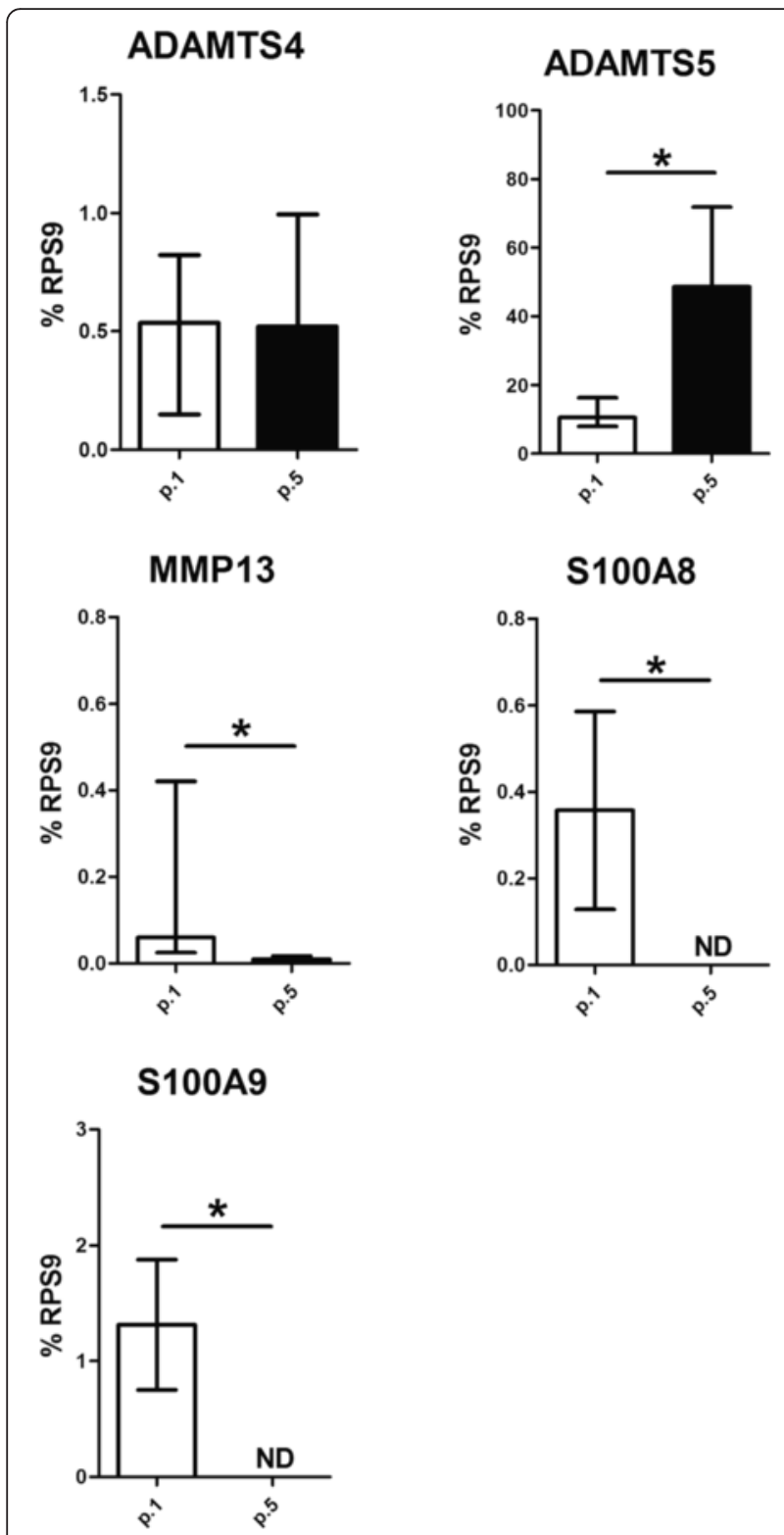

Fig. 4 Evaluation of degradative factors expressed by passage 1 (p.1) and passage 5 (p.5) synovial cells from osteoarthritic moderate-grade synovitis. ADAMTS4, ADAMTS5, MMP13, S100A8, and S100A9 were evaluated in the supernatant of both p.1 and p.5 synovial cells as described in "Methods". Data are expressed as the median and interquartile range $(n=22)$. *Significant differences between $p .1$ and p.5 synovial cells: $p<0.005$. ND not detected

degradative factors, thus creating a significantly different milieu from p.5 synovial cells. Therefore, as p.1 and p.5 synovial cells represent two different cell culture models, we tested whether they could differently affect another cell type in co-culture. We chose adipose stem cells (ASC) as the cell model because they are reportedly activated by an inflammatory environment [22]. We analyzed inflammatory and degradative factors, previously tested in basal conditions in p.1 and p. 5 synovial cells (Figs. 3 and 4), after co-culture with ASC. We did not test IL10 because ASC express and release large amounts of this cytokine, but did not express the other factors analyzed (data not shown). As we previously reported [22], we confirmed that ASC in co-culture with p.1 synovial cells reduced the release of IL6, CXCL8/ IL8, CCL2/MCP-1, CCL3/MIP1 $\alpha$, and CCL5/RANTES (Fig. 5a). Conversely, as shown in Fig. 5b, the co-culture of ASC with p.5 synovial cells (only SF) differently affected the release of the factors evaluated, thus indicating direct dependence by the presence of SM in culture. In particular, ASC when co-cultured with p.5 synovial cells were able to increase the release of IL6 and CXCL8/IL8, however they were unable to affect or significantly decreased, the release of macrophage-like chemokines, such as CCL2/MCP-1 and CCL5/RANTES, respectively. Interestingly, as shown in Fig. 5b, on p.5 synovial cells the ASC were unable to modulate CCL3/MIP1 $\alpha$ that was still not released or expressed (data not shown) by SF. Moreover, as shown in Fig. 5c the analysis of degradative factors revealed that ASC on p.1 synovial cells decreased the expression of ADAMTS5, S100A8, and S100A9, but ADAMTS4 and MMP13 were not affected. Conversely, ASC on p.5 synovial cells induced the expression of MMP13 and did not modulate the other factors analyzed (Fig. 5d).

Moreover, to confirm that the effects observed were directly dependent on the amount of SM in synovial cells p.1 we also evaluated all the inflammatory and degradative factors in p.1 synovial cells isolated from low-grade synovial explants, which, as shown in Fig. 1b, contain a very low percentage of SM (CD68 positive). As shown in Fig. 5e-f, ASC were unable to reduce the inflammatory and degradative factors in p.1 synovial cells from low-grade synovitis, except for CCL5/RANTES and ADAMTS5, but MMP13 was induced.

\section{Immunohistochemical analysis of CD68, CCL3/MIP1 $a$ and S100A8 on synovial tissue}

Furthermore, to confirm that CCL3/MIP1 $\alpha$ and S100A8 were specific markers of SM, we also immunostained serial sections of synovial tissue from patients with moderate synovitis using the positive control macrophage marker CD68. As shown in Fig. 6, we confirmed that SM positive to CD68 were also positive to CCL3/MIP1 $\alpha$ and S100A8, which we also detected only on p.1 synovial cells. Positive cells were mainly located on the lining layer and around the vessels.

\section{Discussion}

Synovitis is a typical feature in a high percentage of patients with OA, even in the early phase of the disease [3]. Hyperplasia in the synovium is associated with an increased number of synovial lining cells in OA, 


\section{Synovial cells from moderate grade synovium}

A

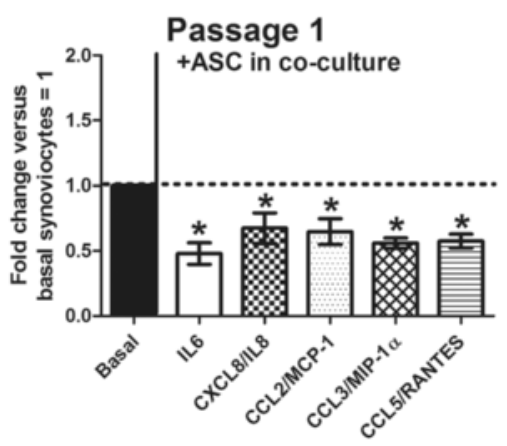

C Passage 1

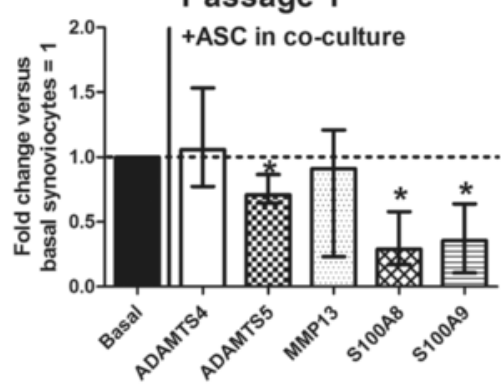

B

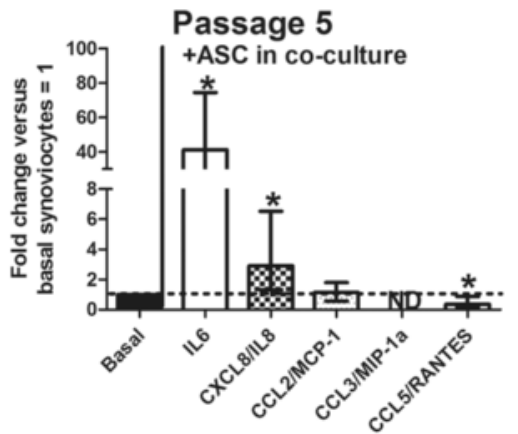

D Passage 5

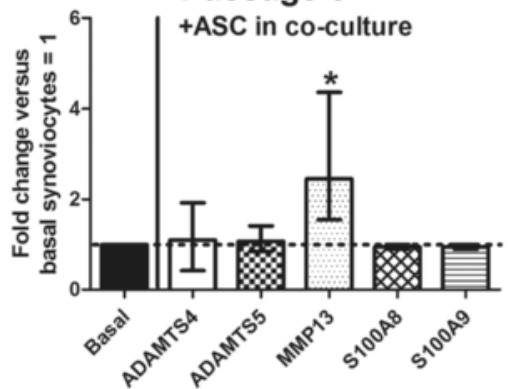

\section{Synovial cells from low grade synovium}

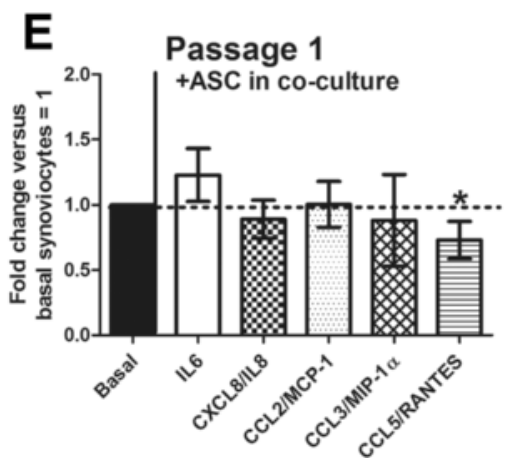

$\mathbf{F}$
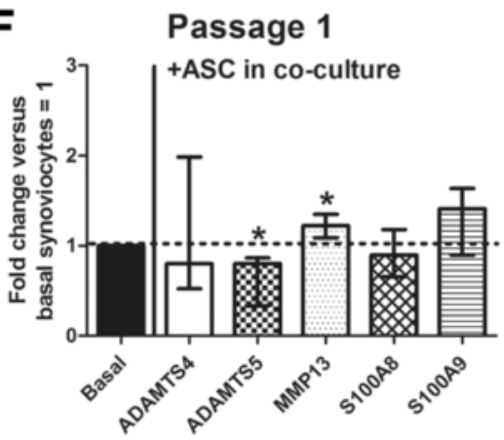

Fig. 5 Evaluation of the role of macrophage in cell co-cultures. a-d Co-culture of adipose stem cells (ASC) with passage 1 (p.1) (a, c) and passage 5 (p.5) (b, d) synovial cells from osteoarthritic moderate-grade synovitis and evaluation of released inflammatory (IL6, CXCL8/IL8, CCL2/MCP-1, CCL3/MIP1a, and CCL5/RANTES) and expressed degradative factors (ADAMTS4, ADAMTS5, MMP13, S100A8, and S100A9). Data are represented as fold changes versus basal synoviocytes $=1$ and expressed as the median and interquartile range $(n=22)$. *Significant differences between p.1 and p.5 synovial cells: $p<0.005$. e-f Co-culture of ASC with p.1 synovial cells from low-grade osteoarthritic synovitis and evaluation of released inflammatory (IL6, CXCL8/L8, CCL2/MCP-1, CCL3/MIP1a, and CCL5/RANTES) (e) and expressed degradative factors (ADAMTS4, ADAMTS5, MMP13, S100A8, and S100A9) (f). Data are represented as fold changes versus basal synoviocytes $=1$ and expressed as the median and interquartile range $(n=4)$. ${ }^{*}$ Significant differences between $p .1$ and p.5 synovial cells: $p<0.005$

accompanied by infiltration of inflammatory cells mainly consisting of macrophages [8]. Synovial tissue is a complex structure mainly composed of SF and SM, and in vitro cell models have mainly focused on SF. Therefore, we characterized synovial tissue from low-grade and moderate-grade synovitis and synovial cell outgrowth from cultured nondigested synovial fragments. These cells were analyzed at two cell passages (p.1, a mix of SF and SM and p.5, only SF) to define their phenotype, inflammatory and degradative factors and their functional role. 


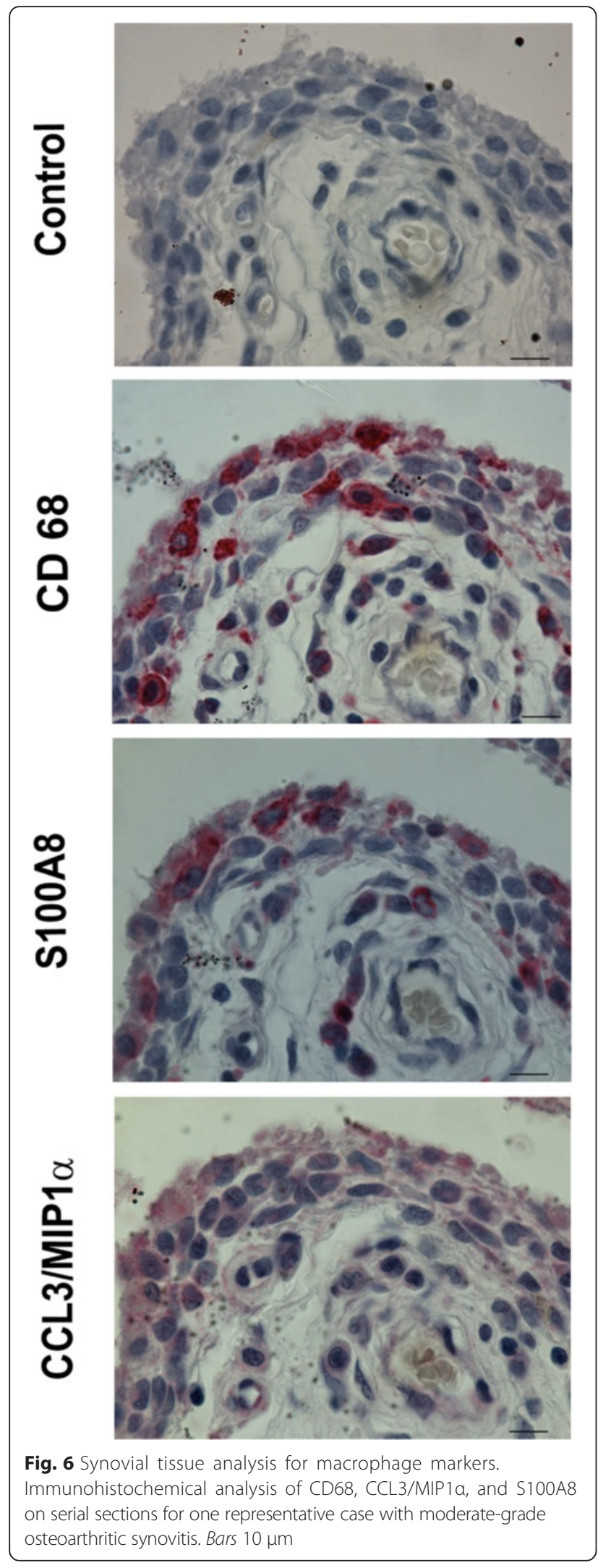

Our data show that synovium from patients with OA and moderate synovitis had approximately $27 \%$ CD68 (SM) and $70 \%$ CD55 (SF) positive cells, which are wellstandardized markers for SM and SF, respectively. The synovial cells outgrowing in culture were evaluated at both p.1 and p.5. SF (p.5) were not positive to typical endothelial (CD31), hematopoietic (CD3, CD34, and CD45) and macrophage-like markers (CD14, CD16, CD68, CD80, and CD163) but they expressed more CD55 and CD106, which are considered relative specific markers of SF [13]. Moreover, SF at both p. 1 and p.5 also expressed increased amounts of CD73, CD90 and CD105, which are considered typical markers for identifying mesenchymal stem cells (MSC). In line with several reports [14], the isolation of MSC and SF from the synovium is mainly based on the adhesion properties of the mononuclear cell fraction in vitro, from which they can be cultured-expanded as SF. However, detailed studies, as recently underlined by De Bari [15], are necessary to determine whether synovium-MSC are SF or different subsets, as in culture they are indistinguishable and no markers permit their selective identification. Only p.1 synovial cells were positive to typical macrophage markers, such as CD14, CD16, and CD68 and to some specific M1 or M2 macrophage markers, such as CD80 and CD163, thus showing the contemporary presence of pro-inflammatory M1 type and anti-inflammatory/ regenerative M2 type in the OA synovium. Moreover, in contrast to other reports, we found the presence of macrophages in p. 1 synovial cell culture, which has been reported to be reduced or absent in synovium-derived cells from digested fragments, and this confirms that enzymatic digestion affects the recovery of the different cell populations present in the synovium $[14,20]$. Interestingly, p.1 synovial cells express only very small amounts of CD31, a typical endothelial marker, thus suggesting that this in vitro procedure does not ensure their isolation.

We found that p.1 synovial cells (mix of SF and SM) release significantly more IL6, CXCL8/IL8, CCL2/MCP-1, CCL3/MIP1 $\alpha$, CCL5/RANTES, and IL10 than those of p.5 (SF), thus suggesting that the absence of SM significantly reduces inflammation and does not induce the anabolic factor IL10. It has been shown that CCL2/MCP1 enhances CD106 [27]; our data show an increased number of positive CD106 on p.5 synovial cells that might be induced by CCL2/MCP1. In fact, even if CCL2/MCP1 is reduced compared to that of p.1 synovial cells, it is released in a greater quantity by p.5 synovial cells.

Bondeson et al. [17] reported that depletion of CD14positive SM was associated with a decline in inflammation associated with decreased IL6, CXCL8/IL8, and CCL2/MCP-1, which is in line with our data in vitro. Among the factors analyzed, we found that S100A8, S100A9, and CCL3/MIP1 $\alpha$ were the only undetectable 
factors (both at molecular and protein level) in p.5 synovial cells, a cell culture characterized by the absence of SM, thus confirming that they are specific markers of SM.

Synovial cells p. 1 and p. 5 represent two different in vitro cell models characterized by the presence and absence of $\mathrm{SM}$, respectively, which are directly responsible for the low- and high-level inflammatory/degradative milieu. Different studies $[28,29]$ have used SF (p.5 synovial cells) as a cell model to test drugs or the effects of other cell types, without taking into consideration that synovial tissue is mainly composed of at least two cell types, SM and SF. It is has been known that OA SM are those mainly responsible for synovial inflammation $[5,16,30]$ and it has been reported that inflammation induces ASC to exert an antiinflammatory effect [22, 31, 32]. Therefore, to better define how the presence or absence of SM in cell culture influences the effects observed we evaluated the effects of ASC on inflammation and degradative factors co-cultured with the synovial p.1 and p.5 cell models. We found that in contrast with the anti-inflammatory effects found on p.1 synovial cells from moderate-grade OA, on p.5 synovial cells the ASC modulated the analyzed inflammatory factors differently. In particular, ASC significantly induced IL6 and CXCL8/IL8, decreased CCL5/RANTES, and did not modulate CCL2/MCP-1 or CCL3/MIP1 $\alpha$, which were still not expressed or released, thus demonstrating and confirming a specific dependence of this chemokine on SM and not on SF. Moreover, it is interesting to note that among the inflammatory factors analyzed, the CCL5/ RANTES chemokine, mainly associated with a signature of synovial inflammation, was the only one that was still down-modulated in the presence of ASC, both in coculture with p.1 with low- or moderate-grade synovitis, and with p.5 synovial cells, thus highlighting that this was an effect independent of the presence of SM.

It has been shown that CCL3/MIP1 $\alpha$ has an important role in the recruitment of infiltrating leukocytes in the arthritic joint. Moreover, the CCL3-null mouse arthritis model is associated with a reduction of infiltrating cells and normal appearance of the synovium and cartilage, and the absence of pannus or bone resorption, thus confirming the important role of this chemokine in OA [33]. This evidence is also corroborated by a report of increased expression and secretion of CCL3-MIP $1 \alpha$ when SF were co-cultured with activated leukocytes (monocytes or polymorphonuclear neutrophils) [34].

Our data show that ASC in co-culture with p.1 synoviocytes from moderate-grade $\mathrm{OA}$ decreased the typical inducible factors ADAMTS5, S100A8, and S100A9, and did not affect ADAMTS4 and MMP13. Conversely, in co-culture with p.5 synovial cells they only induced the expression of MMP13, thus suggesting that in the absence of SM, SF appear to acquire a characteristic more typical of SM, such as increased expression of MMP13. Interestingly, we also found that S100A8 and S100A9, the main catabolic factors produced by activated macrophages, were not detected in p.5 synovial cells co-cultured with ASC, thus confirming their specific expression on $\mathrm{SM}$. These data were also confirmed on moderate-grade OA synovial tissue, where we found that S100A8 and CCL3/MIP1 $\alpha$ were co-expressed with CD68, the typical macrophage marker.

Moreover, we found that S100A8 and S100A9 inhibition in p.1 synovial cells was also associated with IL6 and CXCL8/IL8 inhibition, which, as already reported [35], are strictly dependent. These data are also in line with a recent report that in a murine collagenaseinduced OA model, ASC inhibited synovial activation mainly by reducing S100A8 and S100A9 [36]. Furthermore, large quantities of these catabolic factors have also been found in the synovial tissue of patients with OA, and they predict the development of cartilage destruction [37]. Interestingly, we also confirmed that in contrast to p.1 synovial cells obtained from patients with moderate-grade synovitis, p.1 synovial cells from patients with low-grade synovitis are unable to guide the anti-inflammatory and anti-catabolic effects of ASC, as found for p.5 synovial cells, as SM were present in very small numbers.

\section{Conclusions}

In summary, our data from in vitro analysis show the importance of using the correct in vitro cell models to recreate a milieu that closely resembles OA synovial tissue as the target tissue organ. The availability of in vitro cell models (p.1 and p.5 synovial cells) with large or small numbers of SM effectively reflects the different degrees of OA, which are characterized by different degrees of synovial inflammation, giving the opportunity of testing cells, anti-inflammatory drugs, or factors in a well-defined milieu.

\section{Additional file}

Additional file 1: Immunohistochemical analysis of CD55 and CD68 on representative cases with low grade (left) and moderate grade (right) OA synovitis. Bars $10 \mu \mathrm{m}$. (TIF $2835 \mathrm{~kb}$ )

\footnotetext{
Abbreviations

ADAMTS4: ADAM metallopeptidase with thrombospondin type 1 motif, 4; ADAMTS5: ADAM metallopeptidase with thrombospondin type 1 motif, 5; ASC: adipose stem cells; BSA: bovine serum albumin; CCL2/MCP1: chemokine (C-C motif) ligand 2/monocyte chemotactic protein 1; CCL3/

MIP1a: chemokine (C-C motif) ligand 3/macrophage inflammatory protein 1a; CCL5/RANTES: chemokine (C-C motif) ligand 5; CD: cluster of

differentiation; CXCL8/IL8: chemokine (C-X-C motif) ligand 8/interleukin 8; DMEM: Dulbecco's modified Eagle's medium; GMP: good manufacturing practice; ICAM-1: intracellular adhesion molecule-1; IL10: interleukin 10; IL4: interleukin 4; IL6: interleukin 6; MMP13: matrix metalloproteinase 13; OA: osteoarthritis; PBS: phosphate-buffered saline; PLP: platelet lysate;
} 
RGB: red/green/blue; RPS9: ribosomal protein S9; S100A8: S100 calcium binding protein A8; S100A9: S100 calcium binding protein A9; SF: synovia fibroblast; SM: synovial macrophage; TGF $\beta$ : transforming growth factor- $\beta$; TNFa: tumor necrosis factor-a; VCAM-1: vascular adhesion molecule-1.

\section{Competing interests}

The authors declare that they have no competing interests.

\section{Authors' contributions}

CM conceived and participated in the study design, performing scoring systems, image analyses, interpretation of data, and drafting the article. FP participated in the study design, carried out cell isolation, contributed to data collection and analysis, and helped to revise the manuscript. EG carried out mRNA preparations, conducted real-time PCR experiments, analyzed the data, and revised the manuscript. YS carried out the immunoassays, participated in histological assessment, and revised the manuscript. LG carried out immunohistochemical analysis, analyzed the data, and revised the manuscript. LC carried out flow cytometry analysis, collected the data, and revised the manuscript. GF participated in the provision of study materials, acquired clinical data, obtained written consent from patients, and revised the manuscript. SF-P provided GMP-ASC and revised the manuscript. GL conceived the study, performed statistical analysis, and gave final approval of the manuscript. All authors read and approved the final version of the manuscript.

\section{Acknowledgements}

The authors wish to thank Mr. Keith Smith for editing. This study was partially supported by the European Seventh Framework Programme (project ADIPOA; Health-2009-1.4-3, 241719) and partially by Horizon 2020 (project ADIPOA2; PHC-15-2014, 643809).

\section{Author details}

'SC Laboratorio di Immunoreumatologia e Rigenerazione Tissutale, Istituto Ortopedico Rizzoli, Via di Barbiano 1/10, Bologna 40136, Italy. ${ }^{2}$ SD Laboratorio RAMSES, Istituto Ortopedico Rizzoli, Bologna 40136, Italy. ${ }^{3}$ Clinica Ortopedica e Traumatologica II, Istituto Ortopedico Rizzoli, Bologna 40136, Italy. "EFS-Pyrénéés-Méditerranéé, Toulouse F-31300, France.

\section{Received: 5 November 2015 Accepted: 24 March 2016} Published online: 04 April 2016

\section{References}

1. Loeser RF, Goldring SR, Scanzello CR, Goldring MB. Osteoarthritis: a disease of the joint as an organ. Arthritis Rheum. 2012;64:1697-707.

2. Krasnokutsky S, Attur M, Palmer G, Samuels J, Abramson SB. Current concepts in the pathogenesis of osteoarthritis. Osteoarthritis Cartilage. 2008; 16 Suppl 3:S1-3.

3. Scanzello CR, Goldring SR. The role of synovitis in osteoarthritis pathogenesis. Bone. 2012;51:249-57.

4. Sellam J, Berenbaum F. The role of synovitis in pathophysiology and clinical symptoms of osteoarthritis. Nat Rev Rheumatol. 2010;6:625-35.

5. De Lange-Brokaar BJE, loan-Facsinay A, van Osch GJ, Zuurmond AM, Schoones J, et al. Synovial inflammation, immune cells and their cytokines in osteoarthritis: A review. Osteoarthritis and Cartilage. 2012;20:1484-99.

6. Henrotin $Y$, Lambert C, Richette P. Importance of synovitis in osteoarthritis: Evidence for the use of glycosaminoglycans against synovial inflammation. Semin Arthritis Rheum. 2014;43(5):579-87.

7. Lambert C, Dubuc JE, Montell E, Vergés J, Munaut C, Noël A, et al. Gene expression pattern of cells from inflamed and normal areas of osteoarthritis synovial membrane. Arthritis Rheumatol. 2014;66(4):960-8.

8. Benito MJ, Veale DJ, FitzGerald O, van den Berg WB, Bresnihan B. Synovial tissue inflammation in early and late osteoarthritis. Ann Rheum Dis. 2005;64:1263-7.

9. Goldenberg DL, Egan M, Cohen AS. Inflammatory synovitis in degenerative joint disease. J Rheumatol. 1982;9:204-9.

10. Farahat MN, Yanni G, Poston R, Panayi GS. Cytokine expression in synovial membranes of patients with rheumatoid arthritis and osteoarthritis. Ann Rheum Dis. 1993;52:870-5.

11. Bondeson J, Foxwell B, Brennan F, Feldmann M. Defining therapeutic targets by using adenovirus: blocking NF-kappaB inhibits both inflammatory and destructive mechanisms in rheumatoid synovium but spares antiinflammatory mediators. Proc Natl Acad Sci USA. 1999;96:5668-73.
12. Smith MD. The Normal Synovium. Open Rheumatol J. 2011;5:100-6.

13. Bartok B, Firestein GS. Fibroblast-like synoviocytes: Key effector cells in rheumatoid arthritis. Immunol Rev. 2010;233:233-55.

14. De Bari C, Dell'Accio F, Tylzanowski P, Luyten FP. Multipotent mesenchymal stem cells from adult human synovial membrane. Arthritis Rheum. 2001;44:1928-42.

15. De Bari C. Are mesenchymal stem cells in rheumatoid arthritis the good or bad guys? Arthritis Res Ther. 2015;17(1):113.

16. Bondeson J, Blom AB, Wainwright S, Hughes C, Caterson B, van den Berg WB. The role of synovial macrophages and macrophage-produced mediators in driving inflammatory and destructive responses in osteoarthritis. Arthritis Rheum. 2010;62:647-57.

17. Bondeson J, Wainwright SD, Lauder S, Amos N, Hughes CE. The role of synovial macrophages and macrophage-produced cytokines in driving aggrecanases, matrix metalloproteinases, and other destructive and inflammatory responses in osteoarthritis. Arthritis Res Ther. 2006:8:R187.

18. Scanzello CR, McKeon B, Swaim BH, DiCarlo E, Asomugha EU, Kanda V, et al. Synovial inflammation in patients undergoing arthroscopic meniscectomy: Molecular characterization and relationship to symptoms. Arthritis Rheum. 2011:63:391-400.

19. Tang $\mathrm{CH}, \mathrm{Hsu} \mathrm{CJ}$, Fong YC. The CCL5/CCR5 axis promotes interleukin-6 production in human synovial fibroblasts. Arthritis Rheum. 2010;62:3615-24.

20. Van Landuyt KB, Jones EA, McGonagle D, Luyten FP, Lories RJ. Flow cytometric characterization of freshly isolated and culture expanded human synovial cell populations in patients with chronic arthritis. Arthritis Res Ther. 2010;12:R15.

21. Chang CB, Han SA, Kim EM, Lee S, Seong SC, Lee MC. Chondrogenic potentials of human synovium-derived cells sorted by specific surface markers. Osteoarthritis Cartilage. 2013;21:190-9.

22. Manferdini C, Maumus M, Gabusi E, Piacentini A, Filardo G, Peyrafitte JA, et al. Adipose-derived mesenchymal stem cells exert antiinflammatory effects on chondrocytes and synoviocytes from osteoarthritis patients through prostaglandin E2. Arthritis Rheum. 2013;65:1271-81.

23. Kellgren JH, Lawrence JS. Radiological assessment of osteo-arthrosis. Ann Rheum Dis. 1957;16:494-502.

24. Krenn V, Morawietz L, Häupl T, Neidel J, Petersen I, König A. Grading of chronic synovitis-a histopathological grading system for molecular and diagnostic pathology. Pathol Res Pract. 2002;198:317-25.

25. Lisignoli G, Grassi F, Piacentini A, Cocchini B, Remiddi G, Bevilacqua C, et al. Hyaluronan does not affect cytokine and chemokine expression in osteoarthritic chondrocytes and synoviocytes. Osteoarthritis Cartilage. 2001;9:161-8.

26. Bourin P, Peyrafitte JA, Fleury-Cappellesso S. A first approach for the production of human adipose tissue-derived stromal cells for therapeutic use. Methods Mol Biol. 2011;702:331-43.

27. Lin YM, Hsu CJ, Liao YY, Chou MC, Tang CH. The CCL2/CCR2 axis enhances vascular cell adhesion molecule-1 expression in human synovial fibroblasts. PLoS One. 2012;7, e49999.

28. Eymard F, Pigenet A, Citadelle D, Flouzat-Lachaniette $\mathrm{CH}$, Poignard A, Benelli $C$, et al. Infrapatellar fat pad induces an inflammatory and a prodegradative phenotype in autologous fibroblast-like synoviocytes from patients with knee OA. Arthritis Rheumatol. 2014;66(8):2165-74.

29. Sieghart D, Liszt M, Wanivenhaus A, Bröll H, Kiener H, Klösch B, et al. Hydrogen sulphide decreases IL-1 $\beta$-induced activation of fibroblast-like synoviocytes from patients with osteoarthritis. J Cell Mol Med. 2015;19:187-97.

30. Han SA, Lee S, Seong SC, Lee MC. Effects of CD14 Macrophages and proinflammatory cytokines on chondrogenesis in osteoarthritic synoviumderived stem cells. Tissue Eng Part A. 2014;20:2680-91.

31. ter Huurne M, Schelbergen R, Blattes R, Blom A, de Munter W, Grevers LC, et al. Antiinflammatory and chondroprotective effects of intraarticular injection of adipose-derived stem cells in experimental osteoarthritis. Arthritis Rheum. 2012;64:3604-13

32. Manferdini C, Maumus M, Gabusi E, Paolella F, Grassi F, Jorgensen C, et al. Lack of anti-inflammatory and anti-catabolic effects on basal inflamed osteoarthritic chondrocytes or synoviocytes by adipose stem cellconditioned medium. Osteoarthritis Cartilage. 2015;23(11):2045-57.

33. Chintalacharuvu SR, Wang JX, Giaconia JM, Venkataraman C. An essential role for CCL3 in the development of collagen antibody-induced arthritis. Immunol Lett. 2005;100:202-4.

34. Hanyuda M, Kasama T, Isozaki T, Matsunawa MM, Yajima N, Miyaoka H, et al. Activated leucocytes express and secrete macrophage inflammatory protein-1 upon interaction with synovial fibroblasts of rheumatoid arthritis via a 2-integrin/ICAM-1 mechanism. Rheumatology. 2003;42:1390-7. 
35. Simard JC, Cesaro A, Chapeton-Montes J, Tardif M, Antoine F, Girard D, et al. S100A8 and S100A9 induce cytokine expression and regulate the NLRP3 inflammasome via ROS-dependent activation of NF-kB. PLoS One. 2013;8, e72138.

36. Schelbergen RF, van Dalen S, ter Huurne M, Roth J, Vogl T, Noël D, et al. Treatment efficacy of adipose-derived stem cells in experimental osteoarthritis is driven by high synovial activation and reflected by S100A8/ A9 serum levels. Osteoarthritis Cartilage. 2014;22:1158-66.

37. Schelbergen RF, de Munter W, van den Bosch MH, Lafeber FP, Sloetjes A, Vogl T, et al. Alarmins S100A8/S100A9 aggravate osteophyte formation in experimental osteoarthritis and predict osteophyte progression in early human symptomatic osteoarthritis. Ann Rheum Dis. 2014. doi:10.1136/ annrheumdis-2014-205480

Submit your next manuscript to BioMed Central and we will help you at every step:

- We accept pre-submission inquiries

- Our selector tool helps you to find the most relevant journal

- We provide round the clock customer support

- Convenient online submission

- Thorough peer review

- Inclusion in PubMed and all major indexing services

- Maximum visibility for your research

Submit your manuscript at www.biomedcentral.com/submit 